\title{
News from the San Antonio Breast Cancer Symposium 2015
}

\author{
Chair: $\quad$ Cornelia Liedtke ${ }^{a}$ \\ Participants: Rupert Bartsch ${ }^{\mathrm{b}}$ Hans-Christian Kolberg ${ }^{\mathrm{c}}$ Christian Schem $^{\mathrm{d}} \quad$ Isabell Witzel $^{\mathrm{e}}$ \\ ${ }^{a}$ Konservative Tumortherapie und Studienzentrale, Brustzentrum, Klinik für Frauenheilkunde und Geburtshilfe, Universitätsklinikum \\ Schleswig-Holstein / Campus Lübeck, Lübeck, Germany; \\ b Universitätsklinik für Innere Medizin 1, Klinische Abteilung für Onkologie, Comprehensive Cancer Center, Medizinische Universität Wien, \\ Vienna, Austria; \\ ${ }^{c}$ Klinik für Gynäkologie und Geburtshilfe, Marienhospital Bottrop, Bottrop, Germany; \\ ${ }^{d}$ Klinik für Gynäkologie und Geburtshilfe, Universitätsklinikum Schleswig-Holstein, Campus Kiel, Kiel, Germany;

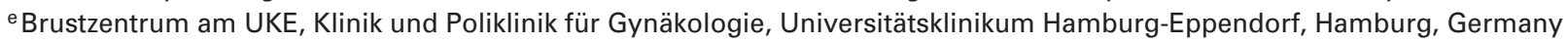

\section{Question 1: Which data presented at this year's San Antonio Breast Cancer Symposium 2015 would you appreciate as representing the most ground-breaking result?}

Bartsch: In my opinion, the most interesting presentations were the ones on the potential role of carboplatin in neoadjuvant treatment of triple-negative breast cancer (TNBC) patients. Updated results of the randomized phase II GeparSixto and CALGB 40603 trials again emphasized that the addition of carboplatin to neoadjuvant chemotherapy significantly improved pathological complete remission (pCR) rates in this population. Somewhat surprisingly, the increased $\mathrm{PCR}$ rate correlated with improved disease-free survival (DFS) and overall survival (OS) in GeparSixto, while - as expected in a phase II study - no such correlation was seen in CALGB 40603. Of note, results of the latter study stressed that patients with residual cancer burden (RCB) score I had an outcome comparable to the outcome of patients with pCR. GeparSixto, again, suggested that the effect of carboplatin was most pronounced in BRCA wild-type patients contradicting conventional wisdom that the addition of carboplatin may be of greatest relevance in patients with $B R C A$ germ-line mutations. These data are well in line with new data from the GeparQuinto study that were also presented; here, it was shown that $\mathrm{pCR}$ rates were generally higher in patients harboring $B R C A$ mutations even if they received non platinum-containing neoadjuvant chemotherapy (50\% vs. $31.1 \% ; \mathrm{p}=0.002$ ). In summary, these results suggest that carboplatin may be added to standard anthracycline/taxane-containing neoadjuvant chemotherapy in high-risk triple-negative patients ir- respective of $B R C A$ mutation status. In order to confirm this assumption, phase III trials are urgently warranted.

Kolberg: While the overall results of the BELLE-2 trial were disappointing with a benefit derived from the addition of the PI3Kinhibitor buparlisib to fulvestrant in metastatic breast cancer (MBC) of less than 2 months increase in progression-free survival (PFS), the study still was one of the highlights of the SABCS 2015. The importance of this presentation is best described by the term 'hidden beauty' in its design. The study group around Jose Baselga planned an analysis on the influence of PIK3CA mutations on the effect of buparlisib, testing the hypothesis that the effect would be more pronounced in mutated patients than in wild-type patients. And, not unexpectedly, this could be shown, with a hazard ratio (HR) in wild-type PIK3CA of 1.05 compared to a HR of 0.56 in patients with PIK3CA mutations, representing a PFS difference of 4.2 months. And although this of course indicates that PIK $3 C A$ mutations are possible predictive biomarkers for the efficacy of PI3K inhibitors, this still is not what makes this presentation so unique. The revolutionary novelty is how the mutations were detected. The PIK3CA mutations have been analyzed from ctDNA samples from the patients' blood and their detection was predictive for the benefit derived from buparlisib. To my knowledge this is the first time the results of a liquid biopsy could define a predictive marker for the therapeutic efficacy of an agent in MBC. This is what I would call a ground-breaking result.

Schem: Ground-breaking and practice-changing results is often a big word. But in my opinion this year's meeting added a few pieces to the puzzle regarding the use of platinum-containing regimens for TNBC patients and/or BRCA1/2 mutation carriers. The

\section{KARGER}

Fax +497614520714
() 2016 S. Karger GmbH, Freiburg

$1661-3791 / 16 / 0111-0074 \$ 39.50 / 0$
PD Dr. med. Cornelia Liedtke

Brustzentrum, Klinik für Frauenheilkunde und Geburtshilfe Universitätsklinikum Schleswig-Holstein / Campus Lübeck Ratzeburger Allee 160, 23538 Lübeck, Germany cornelia.liedtke@ uksh.de 
role of carboplatin seems to be important for patients with TNBC even without BRCA1/2 mutations as presented by von Minckwitz et al. in the early survival analysis of the GeparSixto trial. Contrary to the CALBG/Alliance 40603 trial the GeparSixto trial was not underpowered to demonstrate this benefit. Also the surrogacy of pathologic complete response in TNBC patients was somewhat proven in the presented GeparSixto trial.

Witzel: In my view, the most ground-breaking result was that carboplatin as neoadjuvant agent seems to be effective in all triple-negative patients irrespective of mutation status. In the GeparSixto trial triple-negative patients receiving the combination of paclitaxel, myocet, and carboplatin in a weekly schedule had higher pCR rates than patients without carboplatin (pCR 58.7 vs. $37.9 \%)$. TNBC patients had also a better disease-free survival with carboplatin irrespective of BRCA mutation status (3-year DFS rate 85.8 vs.76.1\%). The CALGB 40603 trial also found that triplenegative patients receiving carboplatin had higher PCR rates but that was not transferred into a better survival. Possible explanations for these differences were the schedule of carboplatin administration (three-weekly vs. weekly) and that the neoadjuvant trials were not set up to detect survival differences but only differences in $\mathrm{pCR}$.

\section{Question 2: Which result do you consider surprising (either in a positive or a negative way)?}

Bartsch: There were several unexpected results presented at this year's San Antonio Breast Cancer Symposium. Perhaps the most surprising ones were data from the Japanese JBCRG-04 trial on adjuvant capecitabine in patients with limited response to prior neoadjuvant chemotherapy. In this study, 910 patients without pCR were randomized to a further 8 cycles of postoperative capecitabine or control. The 2-year DFS rate was $87.3 \%$ in the capecitabine arm as compared to $80.5 \%$ in the observation arm (HR 0.69; $\mathrm{p}=0.001$ ). Of note, a trend towards improved OS was observed as well (2-year OS $96.2 \%$ vs. $93.9 \%$; HR 0.66; $\mathrm{p}=0.086)$. As the curves separated early, it was suggested that this effect may be driven by a reduced recurrence rate in the triple-negative population. In summary, this is the first randomized study to suggest that postneoadjuvant chemotherapy may improve outcome in high-risk patients without pCR. On the other hand, very little information was provided on the important issue what neoadjuvant regimens were administered to these patients. Furthermore, limited activity of capecitabine in TNBC has been suggested in several other studies. Therefore, while interesting, these results do not suffice in order to define a new treatment standard.

Kolberg: The updated results of the GeparSixto trial presented by Gunter von Minckwitz and colleagues showed that the increased pCR rates achieved by the addition of carboplatin to neoadjuvant chemotherapy for TNBC translate into an improvement in DFS and OS, thus reproducing the role of the PCR as a predictive marker also for the use of carboplatin. Surprisingly, the effect of carboplatin was strongest in the BRCA wild-type cohort. Con- sidering the postulated effect of platinum salts on defects in homologous recombination, my expectation was that carboplatin would have a far more pronounced effect on patients with a $B R C A$ germ-line mutation - as seen in other trials, especially in the metastatic setting, e.g. the TNT trial. A possible explanation for this effect might be that the treatment in the GeparSixto trial with a polychemotherapy plus bevacizumab was so effective that the added effect of carboplatin on BRCA germ-line mutated patients 'vanished' and could simply not be shown due to the effectiveness of the co-medication. Based on this possible explanation the findings do not contradict the theory of the efficacy of carboplatin in patients with defects in the homologous recombination like $B R C A$ mutated patients.

Schem: The high expectations on PI3K inhibitors like BKM-120 were put to a rational level. The Belle-2 trial is positive, which proves the biological concept and that's the best news. But the overall advantage presented is small and furthermore, the question which subgroup will benefit the most that will justify the increased rate of toxicity and costs remains unanswered.

Witzel: It was surprising that in a Japanese/Korean trial (Create-X) HER2-negative breast cancer patients had a benefit from the addition of capecitabine if they did not achieve a pCR after neoadjuvant chemotherapy. In a subgroup analysis mainly hormone receptor-negative patients showed this benefit. Although capecitabine was administered in a very high dosage which would add toxicity to current neoadjuvant treatment, the trial could be a basis for new post-neoadjuvant treatment strategies in the group of triple-negative patients with non-pCR who have an impaired prognosis.

\section{Question 3: What is your personal opinion regarding the data presented with regard to prognostic markers - and do you see relevant differences regarding those parameters?}

Bartsch: Interesting information on predictive biomarkers was presented in several studies such as data on PIK3CA mutations in the BELLE- 2 and the neoALLTO trials or data on HER2 status as predictive marker for aromatase inhibitor (AI) versus tamoxifen benefit in a meta-analysis of the ATAC, BIG 1-98, and TEAM trials.

When focusing on prognostic markers, the most pertinent results in my opinion were data on the 10-year risk of disease recurrence as estimated by the EndoPredict (EP) and EPclin (integrating $\mathrm{EP}$ with tumor size and nodal status) scores in patients receiving 5 years of adjuvant endocrine therapy within the ATAC trial and the comparison between the EP score and the OncotypeDX recurrence score (RS). At a median follow-up of nearly 10 years, data from 928 patients suggested that the EP score provided substantially more prognostic information than the RS.

Another interesting study analyzed the prognostic and predictive role of ESR1 mutations. cfDNA analysis was performed in patients treated within the BOLERO-2 trial; results revealed a high rate of Y537S and D538G ESR1 mutations in this population of es- 
trogen receptor (ER)-positive breast cancer patients with prior exposure to a non-steroidal AI. While both mutations were associated with an aggressive course of disease, it is worth mentioning that patients harboring Y537S mutations did apparently not derive any PFS benefit from the addition of everolimus to exemestane (PFS 4.2 vs. 4.1 months; HR 0.98 (0.49-1.94)).

Kolberg: No new prospective data on multigene assays for early breast cancer have been presented this year in San Antonio (except for a poster about TailorX reporting more or less the known data from earlier this year) and most retrospective analyses reported were not practice-changing. A TransATAC project proposed superiority of the EPclin score versus the RS, but the data of this retrospective analysis were not convincing. So unlike the ECC this year, where the first interim analysis of the TailorX trial was presented, the SABCS 2015 did not yield any strikingly new information about this subject. However, one paper presenting 'big data' showed that multigene assays do work in real life. A retrospective analysis from the SEER database collected data from almost 39,000 node-negative breast cancer patients with a median follow-up of 39 months. With an RS below 18 the breast cancerspecific survival was $99.6 \%$, between 18 and 30 still $98.6 \%$ and going down to $95.6 \%$ in the cohort with an RS higher than 30 . Even considering all caveats of a retrospective data collection, the sheer numbers presented in this paper and the concordance of outcomes with RS results are reassuring for physicians using multigene assays in daily decision-making.

Schem: The strongest data concerning prognostic markers presented in San Antonio was the pCR in triple-negative patients in the GeparSixto trial. The long-expected data from the Mindact trial were not presented and would have been very interesting. The comparison of the OncotypeDX test with the EndoPredict test presented by Mitch Dowsett raises a lot of questions. I guess it's clear that adding clinical data to the genetic data set will improve the quality of the prognostic information. I think we have to wait a little longer for the results of all the other ongoing big trials testing genetic predictive gene sets. Up to then we help ourselves with the classical clinical prognostic parameters. But mutational prognostic factors like PIK3CA mutations seem to be relevant, indicating a lower sensitivity to HER2-targeted therapies as outlined by Lajos Pusztai. But the acquired resistance to trastuzumab is a result of different mutations in different genes and each mutation occurs in only few cases. Here we are - personal medicine! Do we have to test for PIK3CA mutations routinely? I guess not yet, because there are so many different ones whose clinical impact is not totally certain.

Witzel: There was quite a lot of data presented on commercially available tests to predict prognosis of breast cancer patients. Michael Dowsett presented a comparison between OncotypeDX and Endopredict as a retrospective analysis in the TransATAC trial. In the ATAC trial postmenopausal women received either anastrozol or tamoxifen as endocrine treatment. In more than 600 of those patients both prognostic tests were performed and compared. Both tests were able to predict patients with a very good prognosis. The Endopredict test performed better when adding tumor size and nodal status - known as the EPclin score. The EPclin score was able to identifiy a group of patients with very good prognosis in nodal-positive breast cancer patients. In conclusion both tests can be used in clinical routine.

\section{Question 4: Which basic science / tumor biological data would you consider as being meaningful for clinical practice - either now or in the future?}

Bartsch: Several interesting - and potentially relevant - basic science and tumor biology data were presented. Personally, I believe that the analysis of long noncoding RNAs performed by Feng et al. may be among the most relevant ones, as this strategy offers the chance for identifying novel prognostic and predictive markers.

Kolberg: Overcoming endocrine resistance is one of the hottest topics in the therapy of especially metastatic breast cancer and the majority of approaches addressing this problem are focusing on the crosstalk between the ER- and the PI3K/Akt/mTOR pathways and the blockade of targets in the latter pathway. This year Ratna Vadlamudi and his group presented a new agent and data derived from cell line experiments using the communication between the ER that is still active even in endocrine resistant tumors (ESR1) and its co-regulators as a target. They developed several ESR1 coregulator binding inhibitors (ECBIs) that showed an expected downregulation of estrogen signaling but also an upregulation of apoptosis and other parameters of cell death. In cell lines with an acquired resistance to tamoxifen and/or letrozole the ECBIs could show a reduction of growth. First experiments in a mouse model proved the oral bioavailability of the agents with minimal side effects. This approach of targeting the estrogen receptor and its coactivators has the potential to play an important role in the treatment of endocrine resistant metastatic breast cancer in the future.

Schem: A bunch of trend-setting abstracts were presented at the 2015 meeting. For example, the group around Thomas Cox used mass spectrometry to identify a new player in bone metastasis. Lysyl oxidase (LOX), which could predict bone metastasis, was found to be released from hypoxic ER-negative cells. This release of LOX appears to lead to the activation of osteoclasts in preparation of the bone for the arrival of tumor cells. Dr. Ellis acknowledged that the group showed that the LOX antibody could suppress bone metastasis in this particular model, possibility advancing treatment or prevention of bone metastasis.

Witzel: Much work was presented regarding immunology. Last year, researchers expressed enormous excitement that great advances in the therapy of breast cancer are near. This year, the quiet consensus among researchers was that research findings were not nearly as striking as they had been led to believe. In patients with advanced ER-positive and HER2-negative breast cancer treated with pembrolizumab, a clinical benefit rate of $20 \%$ ( $5 / 25$ patients) was seen, which seems to be in the range of other breast cancer agents. However, immunotherapy is about to enter clinical practice. 


\section{Question 5: Which developments would you appreciate as being relevant regarding endocrine therapy of breast cancer?}

Bartsch: Results of the BELLE-2 trial were intriguing. In the overall study population of 1,147 patients with hormone receptor-positive / HER2-negative breast cancer refractory to AI, the benefit of adding the pan-class PI3K-inhibitor buparlisib (BKM120) to fulvestrant was mediocre (PFS 6.9 vs. 5.0 months; HR 0.78); a considerably higher toxicity rate was observed in the experimental arm as well. The benefit of adding buparlisib was apparently somewhat more pronounced in patients with activated PI3K pathway status as measured in archival tumor tissue (PFS 6.8 vs. 4.0 months; HR 0.76), but this PFS increase did not meet the preplanned endpoint. On the other hand, PFS in patients with and without PIK3CA mutations in ctDNA revealed distinct PFS patterns: In patients with PIK3CA wild-type ctDNA samples there was no difference in terms of PFS between both groups (HR 1.05) but there was a clear-cut benefit when buparlisib was added to endocrine therapy in the ctDNA PIK3CA mutant population (PFS 7.0 vs. 3.2 months; HR 0.56). While it is too early to draw any final conclusions, this result (and the great $\mathrm{HR}$ difference in between the 2 groups) raises the hope that PIK3CA mutation when analyzed in ctDNA samples may serve as predictive marker for the activity of PI3K inhibitors. Of note, this would be the first novel predictive biomarker in metastatic breast cancer since the introduction of HER2 analysis nearly two decades ago.

Kolberg: The addition of denosumab $60 \mathrm{mg}$ q6m to adjuvant therapy with an $\mathrm{AI}$ in postmenopausal women has been reported earlier to dramatically reduce the fracture rate with a $\mathrm{HR}$ of 0.5 . At the SABCS 2015 Michael Gnant presented additional data concerning the DFS of the 3,425 receptor-positive patients with early breast cancer receiving an $\mathrm{AI}$ and randomized for denosumab dosed as mentioned above or placebo. The range of benefit derived from the addition of denosumab is similar to the effects of adjuvant bisphosphonates and obviously mainly caused by tumors larger than $2 \mathrm{~cm}$, ductal histology and ER- and progesterone receptor (PR)-positivity. The IDMC has recommended an unblinding of the study in 2016. Therefore the following analyses will not be blinded anymore, so the presented data are the final results except for the explorative endpoints to be reported in the future. But still these data define denosumab as a competitor challenging bisphosphonates and particularly simplifying AI therapies for patients who cannot receive bisphosphonates, e.g. because of renal morbidity.

Schem: The treatment-induced fracture rate especially in the adjuvant endocrine treatment with AI was long underestimated. Michael Gnant presented data concerning the fracture rates in the ABCSG18 trial and they were much higher than expected. More importantly these rates were significantly reduced by the use of denosumab with a dose of $60 \mathrm{mg}$ twice yearly. On top there was a strong trend towards an improved DFS for the use of denosumab.

Witzel: The CDK inhibitor palbocliclib has already been approved for the treatment of metastatic breast cancer in the US this year. Several different blockades of pathways regarding the cell cycle are being developed and might help to overcome endocrine resistance. Therefore, in hormone receptor-positive breast cancer, patients can be spared chemotherapies with multiple steps of endocrine treatment that will be available in the near future with only a modest increase in toxicitiy.

\section{Question 6: Was data presented that in your opinion might warrant immediate translation into daily clin- ical routine?}

Schem: I think this question is really difficult. Mostly the data presented confirmed the knowledge which we already had - like the positive impact of T-DM1 as a second-line treatment in HER2-positive $\mathrm{MBC}$, improving OS up to 31 months. Other new data raised more questions but also consolidated the importance of the SABCS meeting in the future. New drugs like CDK4/6 and PI3K inhibitors will be approved in Europe soon, others like the newly hyped PD-L1 antibodies will still have to prove their utility in breast cancer. Preclinical evidence shows that the breast tumor environment may be less conducive to stimulating a cancer-targeted immune response.

Witzel: The 3-year DFS analysis of the ABCSG18 trial was presented by Michael Gnant. 3,425 postmenopausal patients had received an AI and either denosumab (60 mg every 6 months) or placebo as adjuvant treatment. The 3 -year DFS analysis demonstrated that patients receiving denosumab had a lower recurrence rate when the tumor size was more than $2 \mathrm{~cm}$. The first endpoint of the trial had already shown lower fracture rates with denosumab. Therefore, denosumab should be standard of care for patients who receive AI as it was shown to reduce fractures but also might improve survival.

\section{Participants}

Prof. Dr. med. Rupert Bartsch Universitätsklinik für Innere Medizin 1 Klinische Abteilung für Onkologie Comprehensive Cancer Center Medizinische Universität Wien Währinger Gürtel 18-20, Wien 1090, Austria rupert.bartsch@meduniwien.ac.at

Dr. med. Hans-Christian Kolberg Klinik für Gynäkologie und Geburtshilfe Marienhospital Bottrop gGmbH Josef-Albers-Str. 70, 46236 Bottrop, Germany hans-christian.kolberg@mhb-bottrop.de

Priv.-Doz. Dr. med. Christian Schem, MaHM Klinik für Gynäkologie und Geburtshilfe Universitätsklinikum Schleswig-Holstein, Campus Kiel Arnold-Heller-Str. 3, Haus 24, 24105 Kiel, Germany Christian.Schem@uksh.de

PD Dr. med. Isabell Witzel, MPH Brustzentrum am UKE

Klinik und Poliklinik für Gynäkologie Universitätsklinikum Hamburg-Eppendorf Martinistraße 52, 20246 Hamburg, Germany iwitzel@uke.de 